\title{
Sertoli Cells Engineered to Express Insulin to Lower Blood Glucose in Diabetic Mice
}

\author{
Gurvinder Kaur, Lea Ann Thompson, Rachel L. Babcock, Karl Mueller, and Jannette M. Dufour
}

Long-term survival of allo- and xenotransplanted immune-privileged Sertoli cells (SCs) is well documented suggesting that SCs can be used to deliver foreign proteins for cell-based gene therapy. The aim of this study was to use a lentivirus carrying proinsulin cDNA to achieve stable expression and lowering of blood glucose levels (BGLs). A SC line transduced with the lentivirus (MSC-LV-mI) maintained stable insulin expression in vitro. These MSC-LV-mI cells were transplanted and grafts were analyzed for cell survival, continued proinsulin mRNA, and insulin protein expression. All grafts contained MSC-LV-mI cells that expressed proinsulin mRNA and insulin protein. Transplantation of MSC-LV-mI cells into diabetic mice significantly lowered BGLs for 4 days after transplantation. Interestingly, in three transplanted SCID mice and one transplanted BALB/c mouse, the BGLs again significantly lowered by day 50 and 70, respectively. This is the first time SC transduced with a lentiviral vector was able to stably express insulin and lower BGLs. In conclusion, a SC line can be modified to stably express therapeutic proteins (e.g., insulin), thus taking us one step further in the use of SCs as an immune-privileged vehicle for cell-based gene therapy.

Keywords: Sertoli cells, cell-based gene therapy, insulin, lentivirus, transplantation

\section{Introduction}

$\mathbf{S}$ ERTOLI CELLS (SCs) RESIDE within the seminiferous tubules of the testis, where they provide an immunetolerant environment for the developing germ cells. Without this environment, the autogenic germ cells, which develop after the immune system is already established, could elicit an immune response (O'Rand and Romrell, 1977; Yule et al., 1988). SCs create this environment by forming the blood-testis barrier and secreting several immunomodulatory factors (Mital et al., 2010, 2011; Kaur et al., 2014a). These immunoregulatory factors also allow SCs to survive when transplanted across immunological barriers as alloand xenografts without the use of chronic immunosuppressive drugs (Korbutt et al., 1997; Dufour et al., 2003, 2004; Gores et al., 2003; Wright et al., 2016). These unique properties of SCs led to the concept that SCs would make excellent targets as vehicles for the delivery of therapeutic proteins (i.e., cell-based gene therapy). This concept is supported by Dufour et al., and others who have genetically engineered SCs to express foreign proteins (Dufour et al., 2004; Trivedi et al., 2006), including insulin, which requires posttranslational modifications to become biologically ac- tive (Halley et al., 2010; Kaur et al., 2014b; Mital et al., 2014).

Rodent and pig SCs were first engineered to express insulin with an adenoviral vector carrying human proinsulin cDNA (Halley et al., 2010; Mital et al., 2014). The proinsulin transgene was modified with furin (ubiquitously expressed protein convertase) cleavage sites since the nonendocrine SCs do not express the endocrine-specific proprotein convertases necessary to process proinsulin to mature insulin. Normoglycemia was achieved when the genetically engineered SCs were transplanted into diabetic SCID mice. However, the expression of insulin was transient, which is common with adenoviral vectors since they transduce cells through epichromosomal mechanisms (Halley et al., 2010; Mital et al., 2014). Nonetheless, it was demonstrated that SCs are capable of processing and secreting sufficient levels of insulin to transiently normalize blood glucose levels (BGLs).

To demonstrate stable protein expression by SCs, a mouse Sertoli cell line (MSC-1 cells) was transduced with a lentiviral vector containing furin-modified human proinsulin cDNA (MSC-EhI-Zs cells) (Kaur et al., 2014b). The MSCEhI-Zs cells expressed insulin in vitro for $>2$ years (through

\footnotetext{
Department of Cell Biology and Biochemistry, Texas Tech University Health Sciences Center, Lubbock, Texas.
}

(c) Gurvinder Kaur et al., 2018; Published by Mary Ann Liebert, Inc. This Open Access article is distributed under the terms of the Creative Commons Attribution Noncommercial License (http://creativecommons.org/licenses/by-nc/4.0/) which permits any noncommercial use, distribution, and reproduction in any medium, provided the original author(s) and the source are cited. 
several freeze-thaw cycles) and when these cells were transplanted as allografts into diabetic mice with a fully functional immune system, they survived throughout the study (50 days) and maintained their ability to express insulin. Although the MSC-EhI-Zs cells continued to express insulin, the levels of insulin secretion were low and a lowering of BGLs was never observed (Kaur et al., 2014b). Therefore, the focus of this study was to engineer SCs that could stably express insulin at levels, which lower BGL in diabetic mice. MSC-1 cells were transduced with a new modified lentiviral vector carrying a furin-modified mouse proinsulin cDNA (LV-mI). The transduced MSC-1 cells (MSC-LV-mI) were transplanted in both immunocompetent (BALB/c) and immunodeficient (SCID) diabetic mice, and BGLs were measured at different time points throughout the study. The transplanted grafts were also analyzed for survival and continued insulin expression.

\section{Materials and Methods}

\section{Animals}

Eight- to 9-week-old male BALB/c (Charles Rivers Laboratories, Wilmington, MA) or SCID-Beige (Charles Rivers Laboratories) mice were rendered diabetic by an intraperitoneal injection of streptozotocin $(220-230 \mathrm{mg} / \mathrm{kg}$ body weight) (Sigma-Aldrich, St. Louis, MO) 1 week before transplantation. Only those animals exhibiting nonfasting blood glucose values $>20 \mathrm{mM}$ were used as recipients. After transplantation, blood samples obtained from the tail veins of nonfasted recipients were tested by glucose assay at least once a week (OneTouch Ultra; LifeScan). All blood samples were collected between 8:00-8:30 am throughout the study. All animals were maintained under appropriate conditions in accordance with the Institute for Laboratory Animal Research Care and Use of Laboratory Animals and Texas Tech University Health Sciences Center Institutional Animal Care and Use Committee (IACUC)-approved protocols and guidelines of the National Institutes of Health. This study was approved by the IACUC.

\section{Recombinant lentivirus production and transduction}

Mouse proinsulin 2 (Ins2, NCBI Gene ID: 16334) cDNA was modified to allow for processing by furin using optimized codons (OptimumGene ${ }^{\mathrm{TM}}$-Codon Optimizer; GeneScript, Piscataway, NJ), and then created by Origene Technologies, Inc. (Rockville, MD; $\mathrm{mI}$ ). The modified mouse proinsulin 2 construct was subcloned into pIRES2-eGFP plasmid (Clontech Laboratories, Inc., Mountain View, CA). The lentivirus carrying modified mouse proinsulin 2 cDNA and green florescent protein (GFP) under the control of a cytomegalovirus (CMV) promoter (LV-mI; Fig. 1A) was created using the ViraPower ${ }^{\mathrm{TM}}$ Expression System starting with a $p E N T R-2 B$ entry vector recombined into a pLenti 6.3/v5Dest destination vector and packaged and expressed in 293FT cells (Thermo Fisher Scientific, Waltham, MA).

MSC-1 cells were cultured in Dulbecco's modified Eagle's medium (DMEM; Sigma-Aldrich) at $37^{\circ} \mathrm{C}$ and transduced overnight with LV-mI particles (20 MOI/cell). The next day, fresh medium (DMEM containing 5\% [v/v] FBS; Atlanta Biologicals, Flowery branch, GA) was added to the cells. After $60 \mathrm{~h}$, cells were selected for 10 days using $10 \mu \mathrm{g} / \mathrm{mL}$ Blasticidin
(Thermo Fisher Scientific). At this time $100 \%$ of the cells expressed insulin. The stably transduced cells were maintained in DMEM plus $10 \%$ FBS with $1 \mu \mathrm{g} / \mathrm{mL}$ Blasticidin.

\section{RNA extraction and RT-qPCR}

Transduced MSC-1 cells (in vitro) and grafts collected posttransplantation were lysed in $1 \mathrm{~mL}$ of TRIzol reagent (Thermo Fisher Scientific), and total RNA was extracted according to the manufacturer's protocol. The RNA was DNase treated (Thermo Fisher Scientific) and used as a template for oligo (dT)-primed cDNA synthesis, using superscript reverse transcriptase reagent (Thermo Fisher Scientific). PCR was performed for insulin and the housekeeping gene $\beta$-actin, using Go $\mathrm{Taq}^{\circledR}$ Green Master Mix (Promega Corporation, Madison, WI). Insulin and $\beta$-actin were amplified from $50 \mathrm{ng}$ of cDNA under the following PCR conditions: $94^{\circ} \mathrm{C}$ for $2 \mathrm{~min}$; then 35 cycles of $94^{\circ} \mathrm{C}$ for $30 \mathrm{~s}, 57^{\circ} \mathrm{C}$ for $30 \mathrm{~s}$ (insulin) or $58^{\circ} \mathrm{C}$ for $30 \mathrm{~s}$ ( $\beta$-actin), $72^{\circ} \mathrm{C}$ for $30 \mathrm{~s}$; and a final extension of $72^{\circ} \mathrm{C}$ for $10 \mathrm{~min}$. PCR fragments were separated on a $1 \%[\mathrm{w} / \mathrm{v}] \mathrm{TAE}(1 \times \mathrm{TAE}=$ $40 \mathrm{mM}$ Tris-acetate, $1 \mathrm{mM}$ EDTA) agarose gel at $90 \mathrm{~V}$ for $45 \mathrm{~min}$, and fragments were visualized by ethidium bromide staining. All amplicons obtained were of the expected size ( $246 \mathrm{bp}$ for mouse insulin and $500 \mathrm{bp}$ for $\beta$-actin). Primers used were mouse insulin forward, 5'-TCA AGC AGC ACC TTT GT, and reverse, 5'- CAG GTA GAG GGA GCA GAT, and $\beta$-actin forward $5^{\prime}$ - TGT ATT CC CTC CAT CGTG, and reverse, 5'-GGA TCT TCA TGA GGT AGT CTG TC.

For quantification of insulin mRNA, the cDNA was synthesized from 100 ng of RNA using the Superscript VILO ${ }^{\mathrm{TM}}$ Kit (Invitrogen). Real-time PCR was performed using TaqMan Gene expression assay from Applied Biosystems (Thermo Fisher Scientific) (Insulin 2, Assay ID- Mm00731595_gH; and ribosomal 18S, Assay ID-Hs99999901_s1). The real-time PCR was conducted in triplicate for at least three biological samples. Nontemplate controls contained water instead of cDNA. The expression level of the gene of interest was evaluated using the comparative $\mathrm{Ct}$ method. Threshold values $(\mathrm{Ct})$ for the gene of interest and the housekeeping gene $18 \mathrm{~S}$ were determined using QuantStudio $^{\mathrm{TM}}$ 12K Flex software (Applied Biosystems Technology). Ct values for the gene of interest were normalized to $\mathrm{Ct}$ values for $18 \mathrm{~S}$ in each sample and then the fold change for the gene of interest was calculated relative to the level in the reference sample (nontransduced MSC-1 cells).

\section{Immunocytochemistry for insulin}

MSC-LV-mI $\left(1 \times 10^{5}\right.$ cells/well $)$ were cultured on chamber slides in DMEM containing 10\% FBS with $1 \mu \mathrm{g} / \mathrm{mL}$ Blasticidin. After $24 \mathrm{~h}$, cells were fixed with $4 \%$ [w/v] paraformaldehyde (Fisher Scientific, Waltham, MA) and permeabilized with $0.1 \%$ [v/v] Triton X-100 (Fisher Scientific). Slides were blocked with $20 \%$ [v/v] normal goat serum (Vector Laboratories, Burlingame, CA) and incubated with guinea pig polyclonal anti-swine insulin antibody (1:100 dilution; Dako/ Agilent Technologies, Santa Clara, CA). After primary antibody incubation, slides were incubated with Alexa Fluor 594 goat anti-guinea pig (1:400 dilution; Thermo Fisher Scientific) secondary antibody. Slides were then incubated with $4^{\prime}, 6-$ diamidino-2-phenylindole, dihydrochloride (DAPI; $1 \mu \mathrm{g} / \mathrm{mL}$; Molecular Probes, Eugene, OR) to detect cell nuclei. Negative 

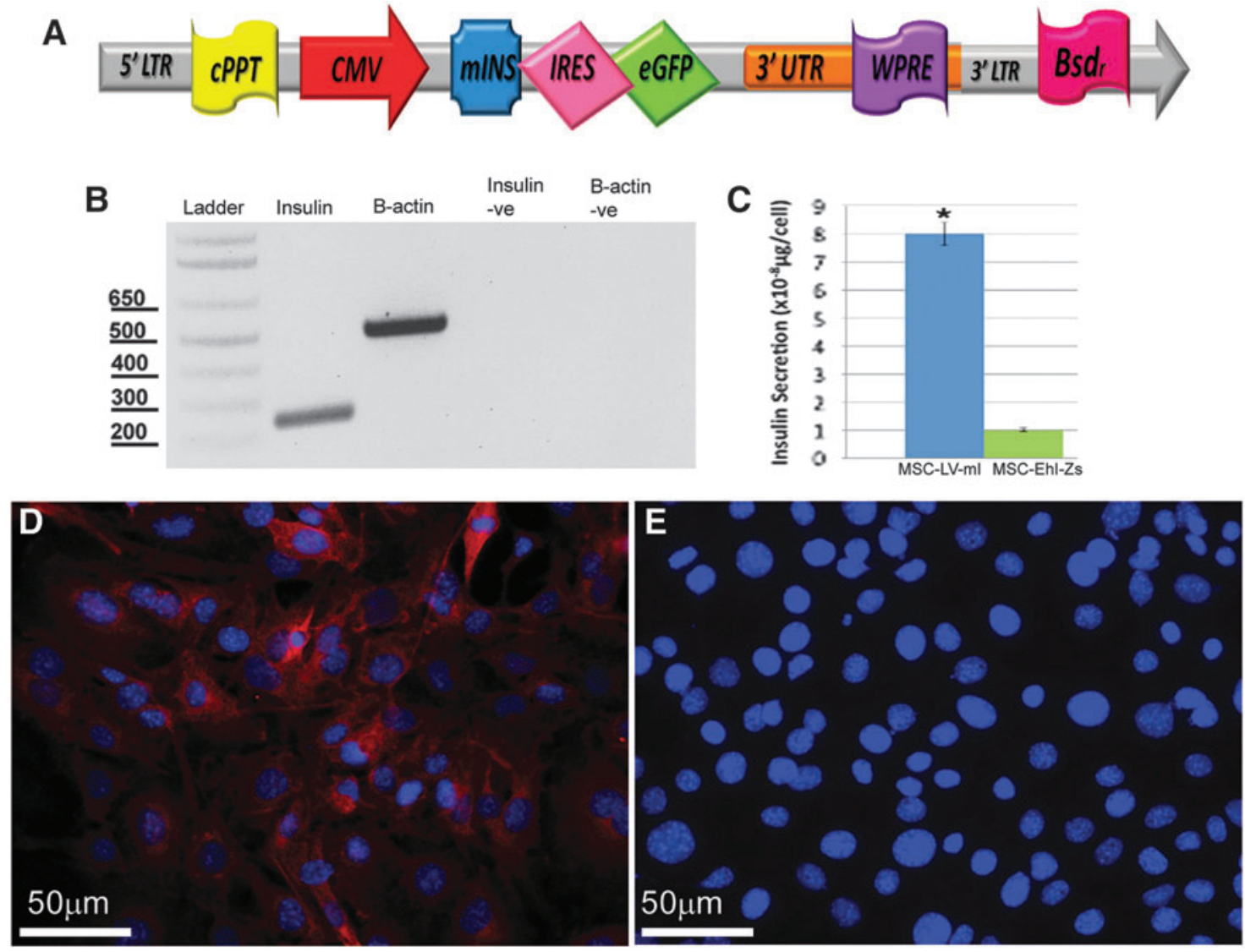

FIG. 1. Mouse Sertoli cells transduced with LV-mI express insulin mRNA and protein. (A) LV-mI, contains a cPPT; CMV promoter; furin-modified mouse proinsulin 2 cDNA (mIns); IRES; eGFP cDNA; WPRE and the Bsd resistance gene. (B) RT-PCR was performed to detect mouse proinsulin mRNA. $\beta$-actin was used as a control. RT negative (-ve) controls containing RNA instead of cDNA was used to rule out genomic DNA contamination. (C) Cell supernatant from MSC-LV$\mathrm{mI}$ and MSC-EhI-Zs cells was collected and ELISA was performed to detect insulin secretion. Data shown are the mean \pm SEM. The asterisk denotes a significant difference in insulin secretion by MSC-LV-mI (blue bar) compared with MSC-EhI-Zs (green bar) cells as determined by unpaired Student's $t$-test $(p \leq 0.05)$. (D) MSC-1 cells transduced with a lentiviral vector containing furin-modified mouse proinsulin cDNA (MSC-LV-mI) were immunostained for insulin (red, D). (E) MSC-LV-mI cells immunostained with secondary antibody were used as negative controls. Cell nuclei were immunostained with DAPI (blue, D and E). Bsd, Blasticidin; CMV, cytomegalovirus; cPPT, central polypurine tract; eGFP, enhanced green fluorescent protein; IRES, internal ribosome entry site; SEM, standard error of the mean; WPRE, Woodchuck Posttranscriptional Regulatory Element.

controls were put through the same procedure without primary antibody. All negative controls lacked a positive reaction. Images were acquired by microscopy (Axio Star plus microscope, using AxioCam MRc digital camera, using AxioVision Rel 4.8 software; Carl Zeiss) and were digitally combined into figures (Photoshop version 7.0 software; Adobe).

\section{Mouse insulin ELISA}

MSC-LV-mI $\left(2.5 \times 10^{5}\right.$ cells/well $)$ were cultured on chamber slides in DMEM containing $10 \%$ FBS with $1 \mu \mathrm{g} /$ $\mathrm{mL}$ Blasticidin. After $48 \mathrm{~h}$, medium was collected and stored at $-80^{\circ} \mathrm{C}$ until analyzed. Samples were analyzed with a human or mouse/rat insulin ELISA following the manufacturer's instructions (EMD Millipore, Billerica, MA).

\section{Transplantation and graft characterization}

Transplantation. Before transplantation, MSC-LV-mI or nontransduced MSC-1 cells were aggregated on nontissue culture-treated Petri dishes containing Ham's F10 medium (Thermo Fisher Scientific) with supplements and 10\% FBS for $48 \mathrm{~h}$ at $37^{\circ} \mathrm{C}$ (Kaur et al., 2014b; Wright et al., 2016). Six or 20 million MSC-LV-mI and 20 million nontransduced MSC-1 cells, calculated using a double-stranded DNA quantitation assay (Quant-iT PicoGreen ${ }^{\circledR}$ ds DNA Assay Kit; Thermo Fisher Scientific), were transplanted into the left renal subcapsular space of isoflurane-anesthetized diabetic BALB/c or SCID-Beige mice as described previously (Halley et al., 2010; Kaur et al., 2014b; Wright et al., 2016). Graft-bearing kidneys were collected over a span of 20-85 days posttransplantation and used for RT-qPCR, immunohistochemistry, or cell culture.

Immunostaining of grafts. Graft-bearing kidneys were immersed in Z-fix (Anatech LTD, Battle Creek, MI), embedded in paraffin, and tissue sections were stained for SV40 large T antigen (MSC-1 cell marker) and insulin as described previously (Kaur et al., 2014b). Sections were 
incubated with monoclonal mouse anti-SV40 large $\mathrm{T}$ antigen (1:100 dilution; BD Biosciences, San Jose, CA) or guinea pig polyclonal anti-swine insulin primary antibodies (1:100 dilution; Dako/Agilent Technologies), and then incubated with biotinylated goat anti-mouse or goat antiguinea pig secondary antibodies (1:200 dilution; Vector Laboratories), respectively. The reaction was developed by incubating the tissue sections with $\mathrm{ABC}$-enzyme complex (Vector Laboratories) followed by Diaminobenzidine (BioGenex, Fremont, CA). The sections were counterstained with Hematoxylin (Sigma-Aldrich). Negative controls were put through the same procedure without primary antibody. All negative controls lacked a positive reaction. Graftbearing kidney tissue served as an additional control as it does not express large $\mathrm{T}$ antigen or insulin. It was negative for large $\mathrm{T}$ antigen and insulin (data not shown). Images were acquired by microscopy as described previously.

Culture of collected graft cells. To obtain single cell suspensions for cell culture, grafts were carefully removed from the kidney, and tissue was chopped gently in Hank's Balanced Salt Solution (HBSS; Sigma-Aldrich). The tissue was incubated with $1 \mathrm{mg} / \mathrm{mL}$ trypsin (Roche Diagnostics, Indianapolis, IN) and $0.4 \mathrm{mg} / \mathrm{mL}$ DNase (Roche Diagnostics) at $37^{\circ} \mathrm{C}$ for $10 \mathrm{~min}$. The dissociated cells were then cultured on chamber slides in DMEM containing 10\% FBS with $1 \mu \mathrm{g} / \mathrm{mL}$ Blasticidin. After 2 days, cells were fixed with $4 \%$ paraformaldehyde, and immunofluorescence was performed as described above (Immunocytochemistry for Insulin).

\section{Statistical analysis}

Data are expressed as the mean \pm standard error of the mean (SEM) or standard deviation (SD) of $n$ independent experiments. Significant differences between two independent groups were calculated by unpaired Student's $t$-test. For multiple comparisons, a one-way analysis of variance (ANOVA) was performed using GraphPad Prism version 4.03 software. When significance was observed, comparison between groups were made using Tukey's post hoc test. A $p$ value of $\leq 0.05$ was considered significant.

\section{Results}

\section{Transduced MSC-1 cells stably secrete insulin in vitro}

MSC-1 cells were transduced with LV-mI (Fig. 1A) and evaluated in vitro for mouse proinsulin mRNA and insulin protein expression as well as insulin secretion levels. The MSC-LV-mI cells expressed proinsulin mRNA and insulin protein demonstrating successful transduction of MSC-1 cells with the LV-mI construct (Fig. 1B and D). The MSCLV-mI cells were a mixed population (i.e., single cell clones were not selected) and therefore the insulin expression was variable within the population. The MSC-LV-mI cells secreted $8 \times 10^{-8} \mu \mathrm{g}$ of insulin per cell when measured by mouse insulin ELISA suggesting that the new vector increased insulin expression eightfold when compared with the previous construct MSC-EhI-Zs, which secreted $1 \times 10^{-8}$ $\mu \mathrm{g} /$ cell (Fig. 1C) (Kaur et al., 2014b). Stable expression of mouse insulin in MSC-LV-mI cells was demonstrated as these cells continued to express insulin in vitro for over 3 years through several freeze-thaw cycles. Nontransduced MSC-1 cells do not express proinsulin mRNA (Figs. 2H, 3J and $4 \mathrm{~J}$ ) or insulin protein in vitro or in vivo (demonstrated previously (Kaur et al., 2014b) and data not shown).

\section{Six million MSC-LV-mI cells transplanted to immunocompetent diabetic mice}

To assess survival and continued expression of insulin by the MSC-LV-mI cells in vivo, 6 million MSC-LV-mI cells were transplanted as allografts into immunocompetent diabetic BALB/c mice $(n=8)$. Nontransduced MSC-1 cells and MSC-EhI-Zs cells were transplanted into diabetic BALB/c $(n=5)$ as controls. BGLs were measured at different time points throughout the study and grafts were collected at day 20 posttransplantation. The collected grafts were immunostained for SV40 large T antigen (marker for MSC-1 cells) to determine cell survival. At day 20, $100 \%(5 / 5)$ of the grafts contained SV40 large T antigen-positive cells (Fig. 2A and "A" in Table 1). Insulin protein expression was detected in $40 \%(2 / 5)$ of the collected MSC-LV-mI grafts (Fig. 2C and " $\mathrm{A}$ " in Table 1). Additionally, cells from the MSC-LV-mI grafts at day 20 were isolated and cultured. Insulin protein expression was observed in $100 \%$ (3/3) of these cultures (Fig. 2E and "A" in Table 1). All nontransduced MSC-1 cell grafts were negative for insulin protein (demonstrated previously by Kaur et al. (2014b). The transplanted MSC-LV-mI cells $(n=4)$ expressed significantly higher amounts of proinsulin mRNA as compared with transplanted nontransduced MSC-1 cells $(n=4)$ (Fig. 2H). In all mice, transplanted with MSC-LV-mI cells, there was a trend $(p=0.056)$ toward lowering of the mean BGL at day 1 compared with pretransplant values (day 0 , Fig. 2G). BGLs in these mice then returned to the diabetic state by day 2 (Fig. 2G). The nontransduced MSC-1 or MSC-EhI-Zs cells had no effect on BGLs (Fig. 2G).

\section{Twenty million MSC-LV-mI cells transplanted in immunodeficient diabetic mice}

To determine whether increasing the number of transplanted cells would result in improved lowering of BGLs, 20 million MSC-LV-mI cells were transplanted in diabetic immunodeficient SCID mice $(n=11)$. Animals transplanted with 20 million nontransduced MSC-1 cells were used as controls $(n=10)$. SCID mice were used to test the construct without interference of effects from an immune response. The nontransduced MSC-1 cells had no effect on the BGLs of diabetic SCID mice (Fig. 3A). Twenty million MSC-LV$\mathrm{mI}$ cells transplanted in the diabetic SCID mice significantly lowered BGLs at day 1 compared with day $0(p=0.02$, Fig. 3A). Interestingly, we observed that the BGLs of three of the mice, transplanted with MSC-LV-mI cells, again significantly decreased by the end of the study (i.e., day 50; Fig. 3B, Supplementary Fig. S1A; Supplementary Data are available online at www.liebertpub.com/dna). Analysis of the BGLs of these three animals revealed that these mice became normoglycemic at day 1 and their BGLs remained significantly decreased for 4 days posttransplantation (Fig. $3 \mathrm{~B}$ ). These animals returned to diabetic state by day 5 and their BGL again started decreasing between days 25 and 46 posttransplantation (Supplementary Fig. S1A). 

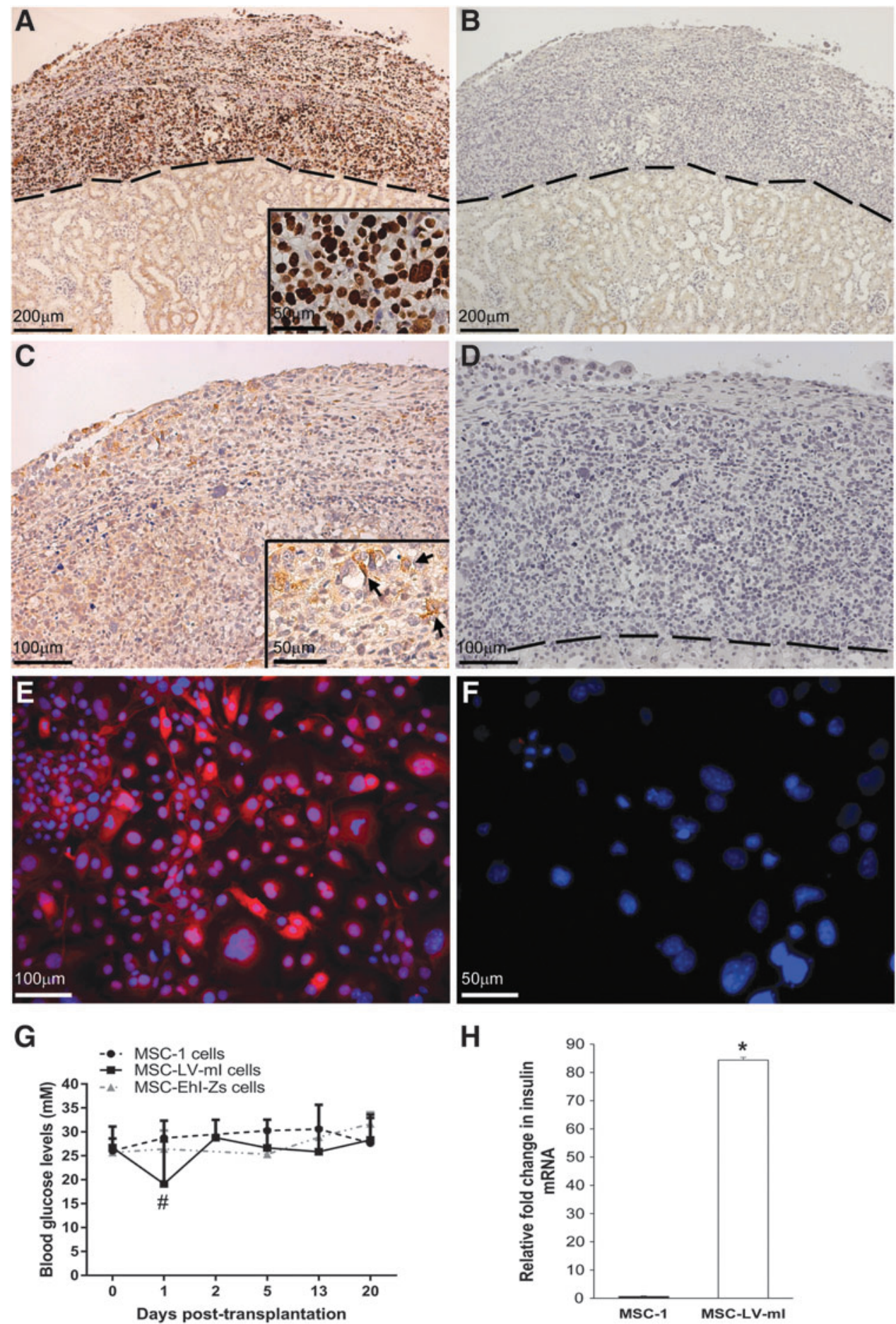

FIG. 2. Six million MSC-LV-mI cells transplanted to BALB/c mice. Six million MSC-LV-mI were transplanted into diabetic BALB/c mice $(n=8)$. (A and $\mathbf{C})$ The graft-bearing kidneys were collected at day 20 posttransplantation and immunostained for large T antigen (MSC-1 cell marker, brown, $\mathbf{A}, n=5$ ) and insulin (brown, $\mathbf{C}, n=5)$. (E) Cells from the MSC-LV-mI grafts were collected at day 20, cultured for 2 days, and immunostained for insulin $($ red, $\mathbf{E}, n=3)$. (G) Average BGLs of animals transplanted with MSC-LV-mI, MSC-EhI-Zs, or nontransduced MSC-1 cells. Bars represent mean \pm SD. Statistical significance of difference versus day 0 was calculated by one-way ANOVA followed by Tukey's post hoc test, ${ }^{\#}$ represents $p=0.056$. (H) RT-qPCR was performed for mouse proinsulin using mRNA isolated from MSC-LV-mI (white bar, $n=4$ ) or nontransduced MSC-1 (black bar, $n=4)$ cell grafts collected from mice 20 days posttransplantation. Data shown are the mean \pm SEM. The asterisk denotes a significant difference in MSC-LV-mI insulin mRNA expression compared with nontransduced MSC-1 cells as determined by unpaired Student's $t$-test $(p \leq 0.05)$. Cell nuclei were counterstained with Hematoxylin (blue, A-D) or DAPI (blue, $\mathbf{E}$ and $\mathbf{F})$. Insets are the high magnification images of (A) and (C). Arrows in the inset $(\mathbf{C})$ indicate insulin-positive MSC-LV-mI cells. (B, D, and F) are negative controls for (A, C, and $\mathbf{E})$, respectively. In (A, B, and $\mathbf{D})$, the dotted line separates the graft (above) from the kidney (below). BGL, blood glucose level; $\mathrm{SD}$, standard deviation; ANOVA, analysis of variance. 

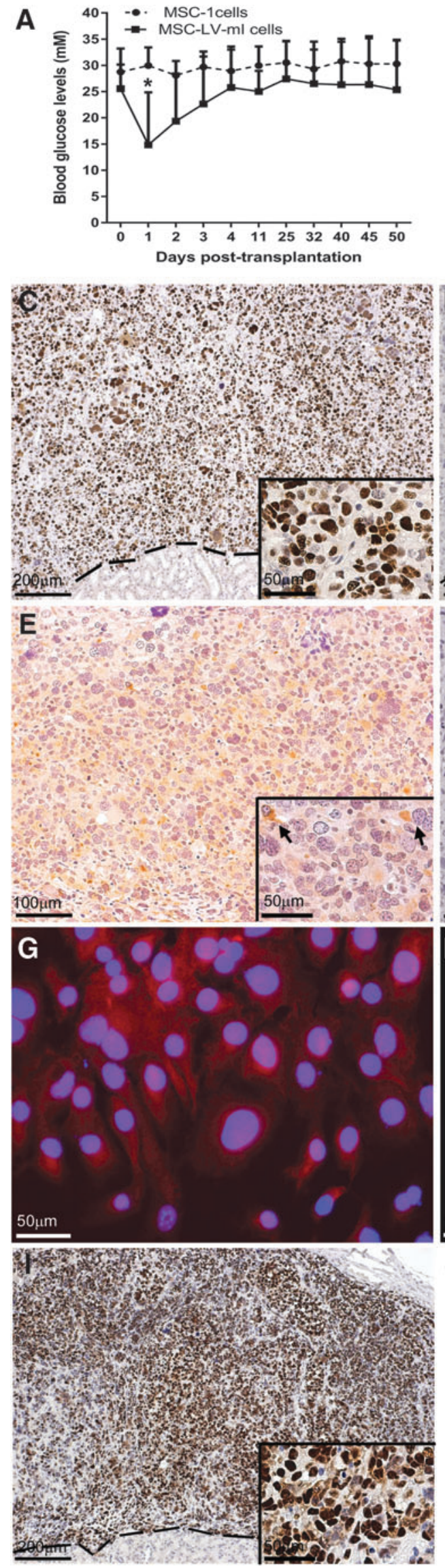

B
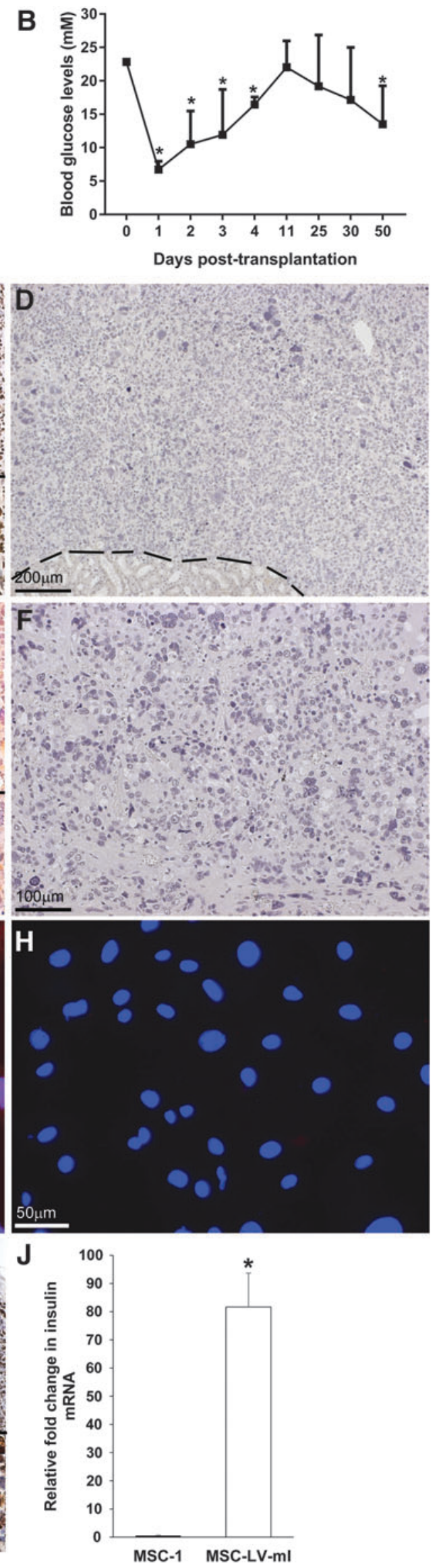

FIG. 3. Twenty million MSC-LV-mI cells transplanted to SCID mice. (A) Twenty million MSC-LV-mI $(n=11)$ or nontransduced MSC-1 $(n=10)$ cells were transplanted underneath the kidney capsule of diabetic SCID mice and BGLs were measured. (B) Data represent the average BGLs of three mice, transplanted with MSC-LV-mI cells, which had decreased BGLs at the end of the study (below $20 \mathrm{mM}$ ). Bars represent mean \pm SD. Statistical significance of difference versus day 0 was calculated by oneway ANOVA followed by Tukey's post hoc test, $*=p \leq 0.05$. (C and I) The MSC-LV-mI (C, $n=6)$ or nontransduced MSC-1 (I, $n=10)$ cell grafts were collected at day 50 posttransplantation and immunostained for large $\mathrm{T}$ antigen (brown). (E) The MSC-LV-mI grafts were immunostained for insulin (brown, $n=6$ ). (G) Cells from the MSC-LV-mI grafts $(n=4)$ were collected, cultured for 2 days, and immunostained for insulin (red). ( J) RT-qPCR was performed for mouse proinsulin using mRNA isolated from MSC-LV-mI (white bar, $n=6)$ or nontransduced MSC-1 (black bar, $n=6$ ) grafts collected at 50 days posttransplantation. Data shown are the mean \pm SEM. The asterisk denotes a significant difference in MSC-LV-mI insulin mRNA expression compared with nontransduced MSC-1 cells as determined by unpaired Student's $t$-test $(p \leq 0.05)$. (D, $\mathbf{F}$, and $\mathbf{H})$ are negative controls for $(\mathbf{C}, \mathbf{E}$, and $\mathbf{G})$, respectively. Cell nuclei were counterstained with Hematoxylin (blue, $\mathbf{C}-\mathbf{F}$ ) or DAPI (blue, $\mathbf{G}$ and $\mathbf{H}$ ). Insets are the high magnification images of (C, E, and I). Arrows in the inset $(\mathbf{E})$ indicate insulinpositive MSC-LV-mI cells. In $(\mathbf{C}, \mathbf{D}$, and $\mathbf{I})$, the dotted line separates the graft (above) from the kidney (below). 
FIG. 4. Twenty million MSC-LV-mI cells transplanted to BALB/c mice. (A) Twenty million MSC-LV-mI $(n=10)$ or nontransduced MSC- 1 cells $(n=3)$ were transplanted underneath the kidney capsule of diabetic $\mathrm{BALB} / \mathrm{c}$ mice and BGLs were measured. (B) Data represent the individual BGLs of three mice, transplanted with MSC-LV-mI cells that had achieved euglycemia at day 1 posttransplantation. Bars represent mean \pm SD. Statistical significance of difference versus day 0 was calculated by one-way ANOVA followed by Tukey's post hoc test. (C and I) The MSC-LV-mI (C, $n=7)$ and nontransduced MSC-1 cell (I, $n=3)$ grafts were collected at day 50 and 70 posttransplantation, respectively, and immunostained for large $\mathrm{T}$ antigen (brown). (E) The MSC-LV-mI grafts were immunostained for insulin (brown, E, $n=7$ ). (G) Cells were isolated from MSC-LV-mI grafts $(n=3)$ at day 50 , cultured for 2 days, and immunostained for insulin (red). ( $\mathbf{J})$ RT-qPCR was performed for mouse proinsulin using mRNA isolated from MSC-LV-mI (white bar, $n=7$ ) or nontransduced MSC1 (black bar, $n=3$ ) grafts collected from mice at 50 and 70 days posttransplantation, respectively. Data shown are the mean \pm SEM. The asterisk denotes a significant difference in MSC-LV-mI insulin mRNA expression compared with nontransduced MSC-1 cells as determined by unpaired Student's $t$-test $(p \leq 0.05)$. (D, F, and $\mathbf{H})$ are negative controls for $(\mathbf{C}, \mathbf{E}$, and $\mathbf{G})$, respectively. Cell nuclei were counterstained with Hematoxylin (blue, C-F) or DAPI (blue, $\mathbf{G}$ and $\mathbf{H})$. Arrows in the inset $(\mathbf{E})$ indicate insulin-positive MSC-LV-mI cells. Insets are the high magnification images of $(\mathbf{C}, \mathbf{E}$, and $\mathbf{I})$. In $(\mathbf{C}, \mathbf{D}$, and I), the dotted line separates the graft (above) from the kidney (below).
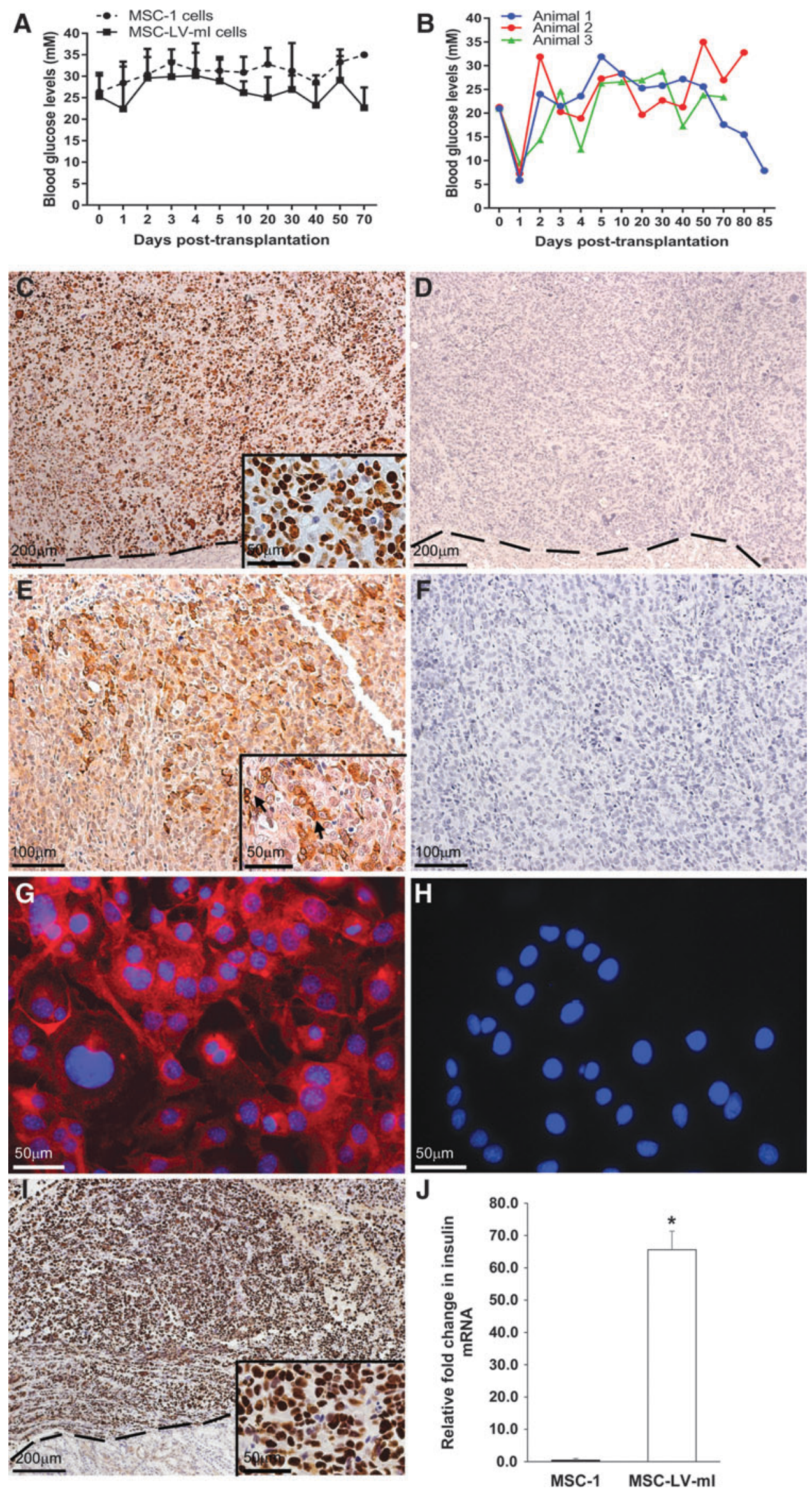
Table 1. Cell Survival and Long-Term Production of Insulin By MSC-LV-mi Cells

A. Transplanted into diabetic BALB/c mice (6 million)

\begin{tabular}{lcc}
\hline & Grafts in vivo $^{\mathrm{a}}$ & ${\text { Isolated } \text { graft cells } \text { in vitro }^{\mathrm{b}}}_{$\cline { 2 - 3 } \text {$Days posttransplantation }}$ \\
\cline { 2 - 3 } Survival $^{\mathrm{c}}$ & Day 20 & Day 20 \\
Insulin protein $^{200 \%(5 / 5)}$ & $100 \%(3 / 3)$ \\
\hline
\end{tabular}

B. Transplanted into diabetic SCID mice (20 million)

\begin{tabular}{lcc}
\hline & Grafts in vivo $^{\mathrm{a}}$ & Isolated graft cells in vitro $^{\mathrm{b}}$ \\
\cline { 2 - 3 } Days posttransplantation & Day 50 & Day 50 \\
\hline Survival $^{\mathrm{c}}$ & $100 \%(6 / 6)$ & $100 \%(4 / 4)$ \\
Insulin protein & $50 \%(3 / 6)$ & $100 \%(4 / 4)$ \\
\hline
\end{tabular}

C. Transplanted into diabetic BALB/c mice (20 million)

\begin{tabular}{|c|c|c|c|c|}
\hline \multirow[b]{2}{*}{ Days posttransplantation } & \multicolumn{2}{|c|}{ Grafts in vivo ${ }^{a}$} & \multicolumn{2}{|c|}{ Isolated graft cells in vitro ${ }^{\mathrm{b}}$} \\
\hline & Day 50 & Day $\geq 70^{\mathrm{d}}$ & Day 50 & Day $\geq 70^{\mathrm{d}}$ \\
\hline $\begin{array}{l}\text { Survival }^{\mathrm{c}} \\
\text { Insulin protein }\end{array}$ & $\begin{array}{l}100 \%(7 / 7) \\
85.7 \%(6 / 7)\end{array}$ & $\begin{array}{l}100 \%(3 / 3) \\
66.6 \%(2 / 3)\end{array}$ & $\begin{array}{l}100 \%(3 / 3) \\
100 \%(3 / 3)\end{array}$ & $\begin{array}{l}100 \%(3 / 3) \\
100 \%(3 / 3)\end{array}$ \\
\hline
\end{tabular}

${ }^{\mathrm{a}}$ Data are presented as the number of positive grafts/total number of grafts.

${ }^{\mathrm{b}}$ Cells were isolated from grafts and percent indicates the number of grafts that contained positive cells.

${ }^{c}$ Survival was determined by SV40 large T antigen immunostaining.

${ }^{\mathrm{d}}$ Grafts were collected at days 70,80 , and 85.

Analysis of the grafts for cell survival revealed that $100 \%$ of the MSC-LV-mI (6/6) and MSC-1 (10/10) cell grafts contained SV40 large T antigen-positive cells at day 50 posttransplantation (Fig. 3C and I and "B" in Table 1). Consistently, proinsulin mRNA was detected at significantly higher levels in MSC-LV-mI grafts $(n=6)$ compared with nontransduced MSC-1 cell grafts $(n=6)$ at day 50 posttransplantation (Fig. 3J). Fifty percent (3/6) of the MSC-LV$\mathrm{mI}$ collected grafts contained insulin-positive cells at day 50 as measured by immunohistochemistry (Fig. 3E and "B" in Table 1). However, when cells were collected and cultured, $100 \%(4 / 4)$ of the grafts contained insulin-positive cells at day 50 posttransplantation (Fig. 3G and "B" in Table 1). No insulin-positive cells were detected in the nontransduced MSC-1 cell grafts.

Twenty million MSC-LV-mI cells transplanted in immunocompetent diabetic mice

To determine the effect of increasing the number of transplanted cells in immunocompetent diabetic mice, 20 million MSC-LV-mI cells (similar to SCID mice) were transplanted into diabetic BALB/c mice $(n=10)$. Grafts were collected at day $50(n=7)$ and at days 70,80 , and 85 . Animals $(n=3)$ transplanted with 20 million nontransduced MSC- 1 cells were used as controls and graft-bearing kidneys were collected at day 70 posttransplantation. On average, no significant lowering of mean BGLs was observed in animals transplanted with MSC-LV-mI or nontransduced MSC-1 cells (Fig. 4A and Supplementary Fig. S2). However, BGLs in three of the mice, transplanted with MSC$\mathrm{LV}-\mathrm{mI}$ cells, were significantly lowered at day 1 and their
BGL continued to fluctuate throughout the study (Fig. 4B). In one mouse, the BGL began to decrease at day 70 and was $7.9 \mathrm{mM}$ when collected at day 85 (Animal 1, Fig. 4B). Analysis of the MSC-LV-mI grafts for cell survival revealed that $100 \%$ of the grafts contained SV40 large T antigenpositive cells at days 50 and $\geq 70$ posttransplantation (Fig. 4C and "C" in Table 1). Similarly, 100\% (3/3) of the MSC-1 cell grafts contained SV40 large T antigen-positive cells at day 70 posttransplantation (Fig. 4I). The proinsulin mRNA was detected at significantly higher levels in MSCLV-mI cell grafts compared with MSC-1 cell grafts (Fig. 4J). When analyzed by immunohistochemistry for insulin, $85.7 \%$ and $66.6 \%$ of the MSC-LV-mI grafts contained insulin-positive cells at days 50 and $\geq 70$, respectively (Fig. 4E and " $\mathrm{C}$ " in Table 1). However, after isolation and culture, $100 \%$ of the grafts contained cells that were insulin positive (Fig. 4G and "C" in Table 1). No insulin-positive cells were detected in nontransduced MSC-1 cell grafts.

\section{Discussion}

Traditional gene therapy has had major setbacks in the past, due to its propensity to cause an immune-mediated "cytokine storm" that is potentially lethal (Raper et al., 2003; Thomas et al., 2003; Kremer et al., 2007; Wang et al., 2009; Limberis et al., 2010; Nayak and Herzog, 2010; Kaur et al., 2012). Furthermore, viral vector-based gene therapy has the potential to cause insertional mutagenesis that can disrupt tumor suppressors or proto-oncogenes, leading to neoplastic changes (Thomas et al., 2003; Montini et al., 2006; Williams, 2009; Nayak and Herzog, 2010; Kaur et al., 2012). The use of immune-privileged SCs as a vehicle for 
cell-based gene therapy could circumvent these issues. For instance, it has been demonstrated that SCs survive without the use of immunosuppressive drugs when transplanted as allo- and xenografts (Korbutt et al., 1997; Dufour et al., 2003, 2004; Gores et al., 2003; Wright et al., 2016). Therefore, SC-based gene therapy is not likely to cause a cytokine storm. Moreover, SCs could also be used to treat autoimmune diseases as they are not targeted and destroyed by the immune response (Suarez-Pinzon et al., 2000; Fallarino et al., 2009), unlike other potential therapies for type 1 diabetes mellitus. Furthermore, SC-based gene therapy has the added advantage in that the cells can be screened for insertional mutagenesis before transplantation, which would avoid neoplastic changes.

To investigate the use of SCs for cell-based gene therapy, it is first critical to demonstrate that SCs can be genetically engineered to express foreign proteins without adversely affecting their immune-privileged abilities. Dufour et al. (2004) demonstrated that GFP-expressing SC isolated from transgenic mice survived and continued to express the foreign protein (GFP) after allotransplantation. Later rat SCs modified to express human neurotrophin-3 (NT-3), produced significant amounts of NT-3 for 3 days after allotransplantation (Trivedi et al., 2006). More recently, using an adenoviral construct carrying a furin-modified human proinsulin transgene, it was verified that primary mouse, rat, and porcine SCs are capable of processing proinsulin and secreting mature insulin at levels that transiently restore normoglycemia in diabetic mice (Halley et al., 2010; Mital et al., 2014). To achieve long-term insulin production, MSC-1 cells were transduced with a lentiviral vector carrying furin-modified human proinsulin cDNA. The MSC-1 cell line was used to test and optimize lentiviral vectors for future use in primary SCs, since the cell line is easier to work with. MSC-1 cells were not clonally selected since they are used to represent primary SCs, where it will be difficult to select clones before transplantation. Stable insulin expression and survival after allotransplantation by transduced MSC-1 cells was attained (Kaur et al., 2014b). However, there was no effect on BGLs with this lentiviral vector.

The aim of this study was to use MSC-1 cells to test a lentiviral vector created to increase insulin secretion to levels that lower BGLs in diabetic mice. To achieve this goal, an enhanced lentiviral vector containing a CMV promoter, a central polypurine tract (cPPT), and a Woodchuck posttranscriptional regulatory element (WPRE) (Fig. 1A) was used. Additionally, a furin-modified mouse proinsulin 2 construct was used instead of the previous human proinsulin construct, which was downstream from an elongation factor 1 alpha promoter. The inclusion of the two cis-acting regulatory elements, cPPT and WPRE, has been reported to enhance the transduction efficiency and transgene expression (Barry et al., 2001; Park and Kay, 2001). For instance, incorporation of a cPPT and posttranscriptional regulatory element into a lentivirus construct increased the transduction efficiency and transgene expression by 5- and 42-fold, respectively, compared with the parent vector (Barry et al., 2001; Powell et al., 2015). Moreover, mouse insulin has been shown to be at least 10 -fold more efficient at binding mouse insulin receptors and when mice are treated with human insulin 20-40 U/kg are needed to normalize BGLs
(Mann et al., 1983; Pepper et al., 2009; Grant et al., 2012). This is in contrast to $0.5-1.0 \mathrm{U} / \mathrm{kg}$ for treatment of humans with diabetes (Christ et al., 1997; Halley et al., 2010). The mouse genome has two genes that express insulin; Ins1 and Ins2. However, the Ins 2 gene is more effective as made evident in a study, where mice containing only Ins 1 had decreased insulin production and developed diabetes, whereas those with only Ins 2 had normal insulin production. The diabetic mice lacking Ins 2 were rescued after the introduction of a transgene encoding for Ins2 (Karaca et al., 2007). Therefore, mouse proinsulin 2 cDNA was used in our study.

To determine if the expression levels were increased with the new lentiviral construct, the amount of insulin secreted by MSC-LV-mI cells in vitro was compared with the amount of insulin secreted by cells transduced with the previous human insulin lentiviral construct (MSC-EhI-Zs) (Kaur et al., 2014b). Additionally, the effect on BGLs after transplantation to diabetic mice was compared. Insulin secretion per cell was increased eightfold with the MSC-LV$\mathrm{mI}$ cells compared with the MSC-EhI-Zs cells (Fig. 1C) (Kaur et al., 2014b). When 6 million MSC-LV-mI cells were transplanted as allografts to diabetic BALB/c mice, a lowering of blood glucose was observed at day 1, whereas BGLs remained within the diabetic range at all times when 6 million MSC-1 cells transduced with the previous human proinsulin lentiviral vector were transplanted as allografts into diabetic $\mathrm{BALB} / \mathrm{c}$ mice. These data indicate that the enhanced lentiviral construct did increase insulin secretion by transduced MSC-1 cells. However, BGLs in mice transplanted with MSC-LV-mI cells returned to the diabetic state by day 2. Nonetheless, this indicates that at the time of transplantation, the MSC-LV-mI cells contained and released enough insulin to lower blood glucose while the previous MSC-EhI-Zs cells did not.

Given the promising results with 6 million MSC-LV-mI cells, the number of cells was increased to 20 million and transplanted into diabetic SCID and BALB/c mice. Immunodeficient diabetic SCID mice were used to remove the variable of an immune response, whereas diabetic BALB/c mice were used to analyze the effect in immunocompetent mice. In SCID mice, overall BGLs at day 1 were significantly lower compared with the pretransplant BGLs (day 0), and in three of the mice transplanted with 20 million cells, BGLs remained significantly lower through day 4 compared with day 1 when 6 million cells were transplanted in immunocompetent $\mathrm{BALB} / \mathrm{c}$ mice. Moreover, in these three mice, BGLs again significantly lowered by day 50 . In contrast, in BALB/c mice, overall BGLs did not change at day 1 but were significantly lowered in three of the mice. As seen with the 6 million cells, the three mice returned to a diabetic state by day 2 . In one mouse, BGLs again decreased between days 70 and 85 . Collectively, this demonstrates that $\mathrm{SC}$ engineered to express insulin can decrease BGLs in diabetic mice.

This is the first time SC transduced with a lentiviral vector were able to stably express insulin and lower BGLs for 4 days after transplantation. The decrease at later time points is suggestive that the transduced MSC-1 cells may have proliferated to reach a critical mass, which secreted enough insulin to lower BGLs. However, not all of the mice achieved normoglycemia. The reason for the variable 
response is not known at this time. One possibility is that the MSC-1 cells were a mixed population that expressed variable levels of insulin. Depending on the number of high expressing cells transplanted and the proliferation rate of these cells, this could have affected BGLs. However, when insulin expression, as measured by RT-qPCR was analyzed, there was no correlation between levels of insulin expression and BGLs. Nevertheless, use of a mixed population of cells is important as it more closely represents primary SCs, which will be necessary in the future.

Interestingly, more SCID mouse transplant recipients were euglycemic than BALB/c mice. The improved lowering of BGLs after transplantation in immunodeficient mice when compared with the immunocompetent mice suggests the immune system is playing a part. The presence of an immune system in BALB/c mice would be constantly targeting the allografted MSC-LV-mI cells as no immunosuppression was used in this study. It is plausible that some of the MSC-LV-mI cells might be rejected by the immune system decreasing the number of cells in the BALB/c mice compared with SCID mice, which might explain the differences in BGLs. It will be interesting to test this construct with primary SCs to see the effect on BGLs.

Future studies will use primary SCs, ideally isolated from transgenic pigs that have been modified with SCs that secrete insulin. The MSC-1 cell line is an ideal resource to optimize vectors for creation of transgenic pigs. To utilize SC-based gene therapy as a sole treatment for type 1 diabetes, further improvements, such as stable lowering of BGLs and glucosedependent release of insulin by SCs, are necessary. However, a combinational therapy that is, SCs secreting basal insulin along with preprandial insulin has the potential to ameliorate diabetes-related complications. For instance, diabetic rats injected with a viral vector carrying rat insulin resulted in insulin production at basal levels thus lowering the BGLs from $>550$ to $\sim 300 \mathrm{mg} / \mathrm{dL}$ (Dong et al., 2002). Addition of commercially available insulin twice daily completely reversed ketotic hyperglycemia, reduced elevated serum fructosamine, glycated hemoglobin (HbA1c), and improved lipid metabolism profiles. Moreover, this therapy significantly reduced advanced glycation end product levels thereby improving the renal function in these animals as demonstrated by correction of proteinuria and albuminuria.

Collectively, our data demonstrate that SCs can be genetically engineered to stably express therapeutic proteins (e.g., insulin) at basal levels thus taking us one step further in the use of SCs as vehicles for cell-based gene therapy. SC-based gene therapy has the potential to be applied to other disorders, where high protein expression and glucose regulation is not mandatory to ameliorate the disease, for instance, protein deficiencies, such as coagulation factors VIII or IX (to treat hemophilia), human erythropoietin (to treat anemia resulting from chronic kidney disease), and dopamine (to treat Parkinson's disease).

\section{Acknowledgments}

This work was supported in part by an NIH grant (HD067400) from the Eunice Kennedy Shriver National Institute of Child Health and Human Development, funding from The Jasper L. and Jack Denton Wilson Foundation, and The Ted Nash Long Life Foundation to J.M.D. L.A.T. is a recipient of an American Heart Association predoctoral fellowship.

\section{Disclosure Statement}

No competing financial interests exist.

\section{References}

Barry, S.C., Harder, B., Brzezinski, M., Flint, L.Y., Seppen, J., and Osborne, W.R. (2001). Lentivirus vectors encoding both central polypurine tract and posttranscriptional regulatory element provide enhanced transduction and transgene expression. Hum Gene Ther 12, 1103-1108.

Christ, M., Lusky, M., Stoeckel, F., Dreyer, D., Dieterle, A., Michou, A.I., et al. (1997). Gene therapy with recombinant adenovirus vectors: evaluation of the host immune response. Immunol Lett 57, 19-25.

Dong, H., Altomonte, J., Morral, N., Meseck, M., Thung, S.N., and Woo, S.L. (2002). Basal insulin gene expression significantly improves conventional insulin therapy in type 1 diabetic rats. Diabetes 51, 130-138.

Dufour, J.M., Hemendinger, R., Halberstadt, C.R., Gores, P., Emerich, D.F., Korbutt, G.S., et al. (2004). Genetically engineered Sertoli cells are able to survive allogeneic transplantation. Gene Ther 11, 694-700.

Dufour, J.M., Rajotte, R.V., Kin, T., and Korbutt, G.S. (2003). Immunoprotection of rat islet xenografts by cotransplantation with sertoli cells and a single injection of antilymphocyte serum. Transplantation 75, 1594-1596.

Fallarino, F., Luca, G., Calvitti, M., Mancuso, F., Nastruzzi, C., Fioretti, M.C., et al. (2009). Therapy of experimental type 1 diabetes by isolated Sertoli cell xenografts alone. J Exp Med 206, 2511-2526.

Gores, P.F., Hayes, D.H., Copeland, M.J., Korbutt, G.S., Halberstadt, C., Kirkpatrick, S.A., et al. (2003). Long-term survival of intratesticular porcine islets in nonimmunosuppressed beagles. Transplantation 75, 613-618.

Grant, C.W., Duclos, S.K., Moran-Paul, C.M., Yahalom, B., Tirabassi, R.S., Arreaza-Rubin, G., et al. (2012). Development of standardized insulin treatment protocols for spontaneous rodent models of type 1 diabetes. Comp Med 62, 381390.

Halley, K., Dyson, E.L., Kaur, G., Mital, P., Uong, P.M., Dass, B., et al. (2010). Delivery of a therapeutic protein by immune-privileged Sertoli cells. Cell Transplant 19, 16451657.

Karaca, M., Durel, B., Languille, L., Lamotte, L., TourrelCuzin, C., Leroux, L., et al. (2007). Transgenic expression of human INS gene in Ins1/Ins2 double knockout mice leads to insulin underproduction and diabetes in some male mice. Front Biosci 12, 1586-1593.

Kaur, G., Long, C.R., and Dufour, J.M. (2012). Genetically engineered immune privileged Sertoli cells: a new road to cell based gene therapy. Spermatogenesis 2, 23-31.

Kaur, G., Thompson, L.A., and Dufour, J.M. (2014a). Sertoli cells-immunological sentinels of spermatogenesis. Semin Cell Dev Biol 30, 36-44.

Kaur, G., Thompson, L.A., Pasham, M., Tessanne, K., Long, C.R., and Dufour, J.M. (2014b). Sustained expression of insulin by a genetically engineered sertoli cell line after allotransplantation in diabetic BALB/c mice. Biol Reprod 90, 109.

Korbutt, G.S., Elliott, J.F., and Rajotte, R.V. (1997). Cotransplantation of allogeneic islets with allogeneic testicular 
cell aggregates allows long-term graft survival without systemic immunosuppression. Diabetes 46, 317-322.

Kremer, K.L., Dunning, K.R., Parsons, D.W., and Anson, D.S. (2007). Gene delivery to airway epithelial cells in vivo: a direct comparison of apical and basolateral transduction strategies using pseudotyped lentivirus vectors. J Gene Med 9, 362-368

Limberis, M.P., Bell, C.L., Heath, J., and Wilson, J.M. (2010). Activation of transgene-specific $\mathrm{T}$ cells following lentivirusmediated gene delivery to mouse lung. Mol Ther 18, 143150.

Mann, N.P., Johnston, D.I., Reeves, W.G., and Murphy, M.A. (1983). Human insulin and porcine insulin in the treatment of diabetic children: comparison of metabolic control and insulin antibody production. Br Med J (Clin Res Ed) 287, 1580 1582.

Mital, P., Hinton, B.T., and Dufour, J.M. (2011). The bloodtestis and blood-epididymis barriers are more than just their tight junctions. Biol Reprod 84, 851-858.

Mital, P., Kaur, G., Bowlin, B., Paniagua, N.J., Korbutt, G.S., and Dufour, J.M. (2014). Nondividing, postpubertal rat sertoli cells resumed proliferation after transplantation. Biol Reprod 90, 13 .

Mital, P., Kaur, G., and Dufour, J.M. (2010). Immunoprotective sertoli cells: making allogeneic and xenogeneic transplantation feasible. Reproduction 139, 495-504.

Montini, E., Cesana, D., Schmidt, M., Sanvito, F., Ponzoni, M., Bartholomae, C., et al. (2006). Hematopoietic stem cell gene transfer in a tumor-prone mouse model uncovers low genotoxicity of lentiviral vector integration. Nat Biotechnol 24, 687-696.

Nayak, S., and Herzog, R.W. (2010). Progress and prospects: immune responses to viral vectors. Gene Ther 17, 295-304.

O'Rand, M.G., and Romrell, L.J. (1977). Appearance of cell surface auto- and isoantigens during spermatogenesis in the rabbit. Dev Biol 55, 347-358.

Park, F., and Kay, M.A. (2001). Modified HIV-1 based lentiviral vectors have an effect on viral transduction efficiency and gene expression in vitro and in vivo. Mol Ther 4, 164 173.

Pepper, A.R., Gall, C., Mazzuca, D.M., Melling, C.W., and White, D.J. (2009). Diabetic rats and mice are resistant to porcine and human insulin: flawed experimental models for testing islet xenografts. Xenotransplantation 16, 502-510.

Powell, S.K., Rivera-Soto, R., and Gray, S.J. (2015). Viral expression cassette elements to enhance transgene target specificity and expression in gene therapy. Discov Med 19, 49-57.
Raper, S.E., Chirmule, N., Lee, F.S., Wivel, N.A., Bagg, A., Gao, G.P., et al. (2003). Fatal systemic inflammatory response syndrome in a ornithine transcarbamylase deficient patient following adenoviral gene transfer. Mol Genet Metab 80, 148-158.

Suarez-Pinzon, W., Korbutt, G.S., Power, R., Hooton, J., Rajotte, R.V., and Rabinovitch, A. (2000). Testicular sertoli cells protect islet beta-cells from autoimmune destruction in NOD mice by a transforming growth factor-beta1-dependent mechanism. Diabetes 49, 1810-1818.

Thomas, C.E., Ehrhardt, A., and Kay, M.A. (2003). Progress and problems with the use of viral vectors for gene therapy. Nat Rev Genet 4, 346-358.

Trivedi, A.A., Igarashi, T., Compagnone, N., Fan, X., Hsu, J.Y., Hall, D.E., et al. (2006). Suitability of allogeneic sertoli cells for ex vivo gene delivery in the injured spinal cord. Exp Neurol 198, 88-100.

Wang, G.P., Levine, B.L., Binder, G.K., Berry, C.C., Malani, N., McGarrity, G., et al. (2009). Analysis of lentiviral vector integration in HIV+ study subjects receiving autologous infusions of gene modified CD4+ T cells. Mol Ther 17, 844850.

Williams, D.A. (2009). Gene therapy continues to mature and to face challenges. Mol Ther 17, 1305-1306.

Wright, K., Dziuk, R., Mital, P., Kaur, G., and Dufour, J.M. (2016). Xenotransplanted pig Sertoli cells inhibit both the alternative and classical pathways of complement-mediated cell lysis while pig islets are killed. Cell Transplant 25, 20272040.

Yule, T.D., Montoya, G.D., Russell, L.D., Williams, T.M., and Tung, K.S. (1988). Autoantigenic germ cells exist outside the blood testis barrier. J Immunol 141, 1161-1167.

Address correspondence to:
Jannette M. Dufour, PhD
Department of Cell Biology and Biochemistry
Texas Tech University Health Sciences Center
36014 th Street
STOP 6540
Lubbock, TX 79430

E-mail: jannette.dufour@ttuhsc.edu

Received for publication August 22, 2017; received in revised form April 23, 2018; accepted May 2, 2018. 